\title{
The methodology for endoluminal irreversible electroporation in porcine models
}

\author{
Tomáš Andrašina ${ }^{1}$, Tomáš Grolich ${ }^{2}$, Michal Crha $^{3}$, Ladislav Stehlík ${ }^{3}$, Tomáš Rohan ${ }^{1}$, \\ Dalibor Červinka ${ }^{4}$, Veronika Novotná ${ }^{4}$,Vladan Bernard ${ }^{5}$, Iva Svobodováa, Vlastimil Válek ${ }^{1}$
}

${ }^{1}$ University Hospital Brno and Masaryk University, Faculty of Medicine,

Department of Radiology and Nuclear Medicine, Brno, Czech Republic

${ }^{2}$ University Hospital Brno and Masaryk University, Faculty of Medicine,

Department of Surgery, Brno, Czech Republic

${ }^{3}$ University of Veterinary and Pharmaceutical Sciences Brno, Faculty of Veterinary Medicine, Department of Surgery and Orthopaedics, Small Animal Clinic, Brno, Czech Republic

${ }^{4}$ Brno University of Technology, Faculty of Electrical Engineering and Communication, Department of Power Electrical and Electronic Engineering, Brno, Czech Republic

${ }^{5}$ Masaryk University, Faculty of Medicine, Department of Biophysics, Brno, Czech Republic

${ }^{6}$ St. Anne's University Hospital Brno and Masaryk University, Faculty of Medicine, First Pathology Department, Brno, Czech Republic

Received February 11, 2019

Accepted April 23, 2019

\begin{abstract}
The aim of this study was to describe the methodology of the surgical technique for endoluminal irreversible electroporation in the biliary tract performed within the perihilar region in porcine models. Endoluminal irreversible electroporation of the common bile duct was performed on eight porcine models using an endoluminal device inserted during laparotomy. The endoluminal device consisted of three electrodes $1 \mathrm{~cm}$ in length, attached at 120 degrees around the balloon catheter. The procedure was conducted with the following parameters: number of pulses 90 , voltage of $1500 \mathrm{~V}$ between each couple of electrodes. Cross sectional imaging and histopathological assessment were employed for evaluations of the ablation zone. Models were sacrificed $24 \mathrm{~h}$ and $96 \mathrm{~h}$ after ablation. The treatment was successful in all porcine models. All animals survived the defined study period. Peri-ablation oedema within the hepatoduodenal ligament and adjacent liver tissue could be measured on post-procedural MRI or CT. Perforation in the site of ablation developed in one model. Histopathological examination showed heavy regressive changes of the ablated tissue. The elastic membranes of the adjacent portal vein were preserved in all models. In our experience, this novel endoluminal modality used within the perihilar region in porcine models is a feasible and well predictable procedure. Further studies should explore the optimal protocol of catheter-based ablation to limit complications.
\end{abstract}

Biliary ablation, magnetic resonance imaging, intraductal ablation, experimental study

Therapeutic procedures used in the care of patients with malignant biliary tract stenosis for the last decades have achieved major progress. Cholangiocarcinoma, most frequent in the elderly population, is difficult to treat due to local extension, limited resectability and high rate of local recurrences in up to $75 \%$ of cases (Serrablo and Tejedor 2013) and make up from $5 \%$ to $30 \%$ of malignant hepatic tumours. Therefore, minimally invasive procedures such as brachytherapy, photodynamic therapy, or endoluminal radiofrequency ablation for tumour treatment are options for those who could not undergo radical surgery or those suffering local recurrence. However, even though a promising theoretical basis for these techniques is set, the clinical results are still far from satisfactory.

The commonly used local ablative method, intraductal radiofrequency ablation, based on the thermal energy destruction, is limited by the "heat sink" effect of blood vessels larger than $3 \mathrm{~mm}$ accompanying the bile duct. This causes insufficient tumour ablation or thermal damage of vessels in the case of more aggressive treatment (Topazian et al. 2013). 
Possible complications of heat are eliminated within irreversible electroporation (IRE), a non-thermal ablation technique where cells are exposed to very short high voltage pulses. High voltage pulses alter the transmembrane electrochemical potential, resulting in cell membrane instabilities and pore formation (Davalos et al. 2005; Rubinsky et al. 2007). When the energy exceeds the potential gradient between the active electrodes, pores of critical diameter are formed, leading to disintegration of the membrane. Damaged cells lose the ability to maintain a stable internal environment and the process leads to cell death. None or minimal thermal effect of technology is preserving the architecture of the intercellular tissue matrix and reparation of tissues along it is possible.

Newly designed and constructed endoluminal catheters with three electrodes for deposition of high voltage pulses were tested in an experimental ex vivo model with thermography measurements (Bernard et al. 2017). The experiments demonstrated the functionality of the system of catheters and the IRE generator, showing that tissue heating is minimal $\left(5 \pm 1{ }^{\circ} \mathrm{C}\right)$. But the events that take place in living organisms associated with reparations at the threshold and suprathreshold damage of phospholipid biomembranes by high voltage pulses cannot be estimated solely by ex vivo modelling. The applicability of catheters for ablations in the bile duct and the effect of the ablation method are directly dependent on the reaction of the living organism involved, and in vivo testing should be the first step for the transition to human medicine.

The aim of this study was to prove the concept of endoluminal IRE in healthy bile ducts of an in vivo porcine model with prototype catheter. The secondary aims were to set a standard protocol for intraoperative endoluminal IRE ablations and to set a standard preprocedural and post-procedural evaluation of animal models undergoing the endoluminal IRE procedure in the biliary tree.

\section{Materials and Methods}

Experiments were performed under the supervision of the Department of Surgery of the Faculty of Veterinary Medicine, University of Veterinary and Pharmaceutical Sciences in Brno, Czech Republic, with a prior approval for the use of animal test subjects from the Ethics Committee of the University of Veterinary and Pharmaceutical Sciences in Brno, Czech Republic, according to law on the protection of animals against cruelty (as amended by $\S 17$, paragraph $15 \mathrm{~g}$, Act no. 246/1992 Coll.).

Animal models and general anaesthesia

Eight laboratory female piglets (50\% Duroc, 25\% Pietrain, and 25\% Landrace cross-breed; the median weight $37.5 \mathrm{~kg}$, weight range from 36 to $39 \mathrm{~kg}$ ) were split into two groups of four pigs according to the desired survival time after surgery $(24 \mathrm{~h}$ vs. $96 \mathrm{~h})$. The whole procedure was carried out under general anaesthesia with deep muscle relaxation, and the animals were intubated and artificially ventilated. The following combinations of substances were applied for general anaesthesia. Premedication was performed by application of tiletamine $2 \mathrm{mg} / \mathrm{kg}$ and zolazepam $2 \mathrm{mg} / \mathrm{kg}$ (Zoletil 100, Virbac, Czech Republic), ketamine $2 \mathrm{mg} / \mathrm{kg}$ (Narketan, Vetoquinol, Czech Republic) and xylazine $2 \mathrm{mg} / \mathrm{kg}$ (Sedazine, Fort Dodge, USA) intramuscularly. Lateral ear vein was used for catheterization and propofol at a dose of 1-2 $\mathrm{mg} / \mathrm{kg}$ (Norofol, FreseniusKabi AG, Germany) was given intravenously following by the intubation of all pigs. General anaesthesia was maintained using inhalation of isoflurane (Aerrane, Baxter, USA) and oxygen-air mixture. During anaesthesia, the heart rate, respiratory rate, saturation of peripheral oxygen and capnography were monitored and recorded. Atracrium $(1.5 \mathrm{mg} / \mathrm{kg}$, Tracurium, GlaxoSmithKline, Italy) was injected intravenously for muscle relaxation.

Technology and protocol for irreversible electroporation

The IRE was performed with an experimental balloon catheter, a prototype developed with the support of the University Hospital Brno start-up grant no. 12/13 and with the institutional support of the Ministry of Health. The balloon catheter was made of a heat-resistant balloon catheter with applied conductor (gold compound), which was conductively connected to a generator with micro conductor (silver compound) (Zemlicka Development and Production of Implants, Omice, Czech Republic) (Plate IV, Fig.1). High-voltage pulse generator, which was designed by Department of Power Electrical and Electronic Engineering, Faculty of Electrical Engineering and Communication, Brno University of Technology, allows manual adjustment of power and ablation time (0-3000 V, up to $100 \mu$ s pulses, max amperage $50 \mathrm{~A})$. 
Surgical procedure

An upper midline laparotomy was performed. After exposure of the common bile duct, the short distal part was prepared, and common bile duct puncture followed. The guidewire $(0.035 \mathrm{inch})$ was introduced via puncture needle to the intrahepatic biliary tract, the tract was dilated with a $10 \mathrm{~F}$ dilatator, and then a balloon catheter-electrode was introduced through the $10 \mathrm{~F}$ sheath, reaching the target area of the hepatic hilus (Plate IV Fig. 2, Plate V, Fig. 3). The balloon was inflated with the fluid to the pressure of 1-2 Atm, to achieve an optimal electrode contact with the wall of the bile duct, and IRE was performed according to the protocol. Post-procedurally, the puncture wound in the common bile duct was closed by one stitch with 5/0 Monoplus. Laparotomy was closed with monofilament suture - peritoneum with 1/0 polyglactine (Novosyn, BBraun, Melsungen, Germany), fascia and subcutaneous tissue separately with 1/0 polydioxanone (Monoplus, BBraun) and skin with $3 / 0$ polyamide (Dafilon, BBraun).

\section{IRE protocol}

High electric pulses were delivered in three sets between each pair of electrodes; the third one remained inactive. The ablation protocol was performed using clinically relevant parameters previously validated in experimental studies (Appelbaum et al. 2013; Ben-David et al. 2012). These included: voltage of $1500 \mathrm{~V}$, pulse length of $100 \mu \mathrm{s}, 90$ pulses at a frequency of $1 \mathrm{~Hz}$. The endoluminal catheter was based on an inflatable balloon of $8 \mathrm{~mm}$ diameter, which meant the distance between the active electrodes was $8.4 \mathrm{~mm}(\pi \times \mathrm{d} / 3)$, and the active part of each electrode was $10 \mathrm{~mm}$.

No complications were observed either during anaesthesia or any of the ablation procedures (Plate V, Fig. 4).

Follow-up

After surgery, the animals were awakened and pain was efficiently controlled by meloxicam $(0.2 \mathrm{mg} / \mathrm{kg}$, Metacam $5 \mathrm{mg} / \mathrm{ml}$, Boehringer Ingelheim, Germany) and by i.m. application of Buprenorphine (dosage $0.01 \mathrm{mg} / \mathrm{kg}$, Temgesic, RB Pharmaceuticals Limited, UK). The animals were housed in stone hutches under controlled environmental conditions ( 20 to $22{ }^{\circ} \mathrm{C}$ room temperature, $50 \%$ to $60 \%$ relative humidity, $12 \mathrm{~h}$ light and $12 \mathrm{~h}$ dark cycle).

The effect of ablation was controlled at $2 \mathrm{~h}$ and at $24 \mathrm{~h}$ or $72 \mathrm{~h}$ after the procedure, using computed tomography (CT, GE Healthcare, USA, native examination, arterial and portal-venous phase after i.v. contrast injection) or magnetic resonance imaging (MRI, GE Healthcare, USA, fat saturated 2D FIESTA in two planes, T2 FRFSE in axial plane, T1 native and after i.v. contrast injection, LAVA multiphase in axial plane, thin and thick slab magnetic resonance cholangiopancreatography).

Blood tests to determine the contents of alanine transaminase (ALT), aspartate transaminase (AST), alkaline phosphatase (ALP), gamma-glutamyltransferase (GGT), serum creatinine, the complete blood count and bilirubin were carried out before the operation and $6 \mathrm{~h}, 24 \mathrm{~h}$ or $96 \mathrm{~h}$ after the procedure (clinical chemistry analyser: Abbott Architect c4000, Abbott Laboratories, IL, USA, haematology analyser: Sysmex XT-2000iV, Sysmex Corporation, Kobe, Japan). The entire study population was euthanized at the desired time-point of 24 or $96 \mathrm{~h}$ after the surgery (Thiopental, T61 Intervet International GmbH, Germany). In each porcine model, an en bloc specimen of liver and duodenobiliary complex was excised. Representative tissue samples of each treated area underwent histopathological analysis using haematoxylin and eosin, and orcein staining.

\section{Results}

The balloon insertion and IRE treatment was successful in all porcine models. The $1500 \mathrm{~V}$ and 90 pulses led to a measured ablation current of 4-11 A (mean 7.9 A). All animals survived for the defined study period. The $\mathrm{CT}$ and MR examinations revealed oedema in the hepatoduodenal ligament (market hypodensity on CT imaging, T2 hyperintensity in MRI) (Plate VI, Fig. 5) whereas vascular structures (hepatic artery, portal vein) were patent immediately after the procedure at $24 \mathrm{~h}$ and at $96 \mathrm{~h}$ (Plate VI, Fig. 6). Magnetic resonance cholangiopancreatography visualised the site of IRE ablation as a minor tapering of the common bile duct, probably due to oedema of the surrounding tissue (Plate VII, Fig. 7). Post-contrast imaging showed faint contrast enhancement of the common bile duct wall at the site of ablation and regular enhancement of adjacent vessels (Plate VII, Fig. 8).

Analyses of blood tests demonstrated a normal range in all values of serum bilirubin (at all time intervals in the range of $0.4-14.3 \mu \mathrm{mol} / \mathrm{l})$, creatinine level $(115.4-170.0 \mu \mathrm{mol} / \mathrm{l}$ ), GGT $(0.45-0.99 \mu \mathrm{kat} / \mathrm{l})$, and ALT (0.19-0.99 $\mu \mathrm{kat} / \mathrm{l})$. At the $24 \mathrm{~h}$ time-point, AST levels were elevated in 3 porcine models $(1.84-2.47 \mu \mathrm{kat} / \mathrm{l})$, while two were asymptomatic at $24 \mathrm{~h}$ and one had covered perforation of the main hepatic duct with bilioma at autopsy. Elevation of white blood cells count was reported in 4/8 porcine models $\left(21.3-29.3 \times 10^{9} / 1\right)$ at $24 \mathrm{~h}$ after surgery. 
Macroscopically, the site of ablation was clearly visible in all models at autopsy. At $24 \mathrm{~h}$, haemorrhagic transformation of the ablation zone was observed (Plate VIII, Fig. 9), and in $96 \mathrm{~h}$ turned to yellow.

In one model, bilioma was found in the autopsy due to previously mentioned perforation $(1 / 8)$ (Plate VIII, Fig. 10). Colour changes in the portal vein wall proximal to the site of ablation were macroscopically determined only in one model $(1 / 8)$.

Histopathological examination showed heavy regressive changes of the proximal common bile duct and close vicinity of the adipose tissue, with a depth of changes of 3-12 mm (mean $6 \mathrm{~mm}$ ) (Plate IX, Fig. 11). Mostly the elastic fibres of the bile duct wall were preserved, but in one case, total focal necrosis of the wall was noted with necrosis of the surrounding adipose tissue.

The elastic membranes of the portal vein were preserved while endothelial cells were necrotic in orcein staining (Plate IX, Fig. 12). Similarly, regressive changes were noted in the arterial wall, endothelial cell necrosis, but preserved internal elastic membrane due to non-thermal energy damage of solely plasmatic membranes.

\section{Discussion}

To achieve a long-term local control of tumours of the biliary tract, the technology of ablation is necessary, as it can be performed repeatedly, has a predictable ablation volume due to the absence of heat cooling by adjacent vessels, and has a low risk of injury to these vascular structures. High voltage electrical pulses in IRE, unlike thermal ablation methods, do not damage the connective tissue. Another advantage of IRE is a very sharp zone between damaged and preserved tissues; the more distant peripheral rim is reversibly electroporated, and cell membranes regain their stability (Lee et al. 2007). Although IRE is commercially available, the only application method is the use of $19 \mathrm{G}$ electrodes unsuitable for intraluminal use. Initial experiments with endovascular methods using a balloon catheter were performed by Maor and Rubinsky (2010). The first experimental endoluminal ablation technique with a self-made rigid electrode inserted endorectally was performed by Srimathveeravalli et al. (2013). More recently, their system with non-expandable catheter-based IRE ablation was tested on the porcine ureter and common bile duct (Srimathveeravalli et al. 2015; Ueshima et al. 2018). To our knowledge, our system of expandable electrodes for IRE which benefits from the optimal contact to the walls of tubular structures, was not previously reported in literature. Surgical technique of catheter insertion and direct visualisation of the ablation site was optimal for experimental use, however the system of IRE with endoluminal catheter is applicable for percutaneous or endoscopical approach.

The threshold for irreversible electroporation is the potential gradient between the active electrodes in the range of 500-1000 V/cm (Davalos et al. 2005). Our experimental setting of IRE voltage during ablation of $1500 \mathrm{~V}$ led to a gradient of $1790 \mathrm{~V} / \mathrm{cm}$ between the active electrode attached to a $8 \mathrm{~mm}$ diameter balloon. Even remarkable zones of peribilary ablation zones were reached; this energy setting significantly exceeds the limits of IRE and should cause substantial heating in contact of electrodes with tissue. This possible damage should be the explanation of the common bile duct perforation found in one tested animal. Protocols of lower energy setting in future works may shed more light on this issue.

The imaging studies used in our study clearly show oedema in the hepatoduodenal ligament and should be used for evaluation of the patency of vascular structures. A prior study verified the correlation between the zone of hyperintensity and the extent of ablations in liver parenchyma (Zhang et al. 2010). In our experience, MRI should be used even for the response prediction of ablation in structures in the subhepatic space.

In conclusion, the experiment tested a new prototype of endoluminal balloon catheter, 
through which it will be possible to perform the non-thermal ablation, irreversible electroporation. The selected protocol achieved an observable zone of ablation accompanied with tissue oedema measurable by imaging methods. In our experience, this novel endoluminal modality used within the perihilar region in porcine models is a feasible and well predictable procedure.

\section{Acknowledgements}

This study was supported by the Ministry of Health of the Czech Republic, grant no. 15-32484a and supported by funds from the Faculty of Medicine MU to junior researcher Tomas Andrasina.

\section{References}

Appelbaum L, Ben-David E, Faroja M, Nissenbaum Y, Sosna J, Goldberg SN 2013: Irreversible electroporation ablation: creation of large-volume ablation zones in in vivo porcine liver with four-electrode arrays. Radiology 270: 416-424

Ben-David E, Appelbaum L, Sosna J, Nissenbaum I, Goldberg SN 2012: Characterization of irreversible electroporation ablation in in vivo porcine liver. Am J Roentgenol 198: W62-W68.

Bernard V, Andrašina T, Červinka D, Martiš J, Procházka P, Mornstein V, Válek V 2017: A thermographic comparison of irreversible electroporation and radiofrequency ablation. IRBM 38: 26-33

Davalos RV, Mir ILM, Rubinsky B 2005: Tissue ablation with irreversible electroporation. Ann Biomed En. 33: 223-231

Lee EW, Loh CT, Kee ST 2007: Imaging guided percutaneous irreversible electroporation: ultrasound and immunohistological correlation. Technol Cancer Res Treat 6: 287-294

Maor E, Rubinsky B 2010: Endovascular nonthermal irreversible electroporation: a finite element analysis. J Biomech Eng 132: 031008

Rubinsky B, Onik G, Mikus P 2007 : Irreversible electroporation: a new ablation modality--clinical implications. Technol Cancer Res Treat 6: 37-48

Serrablo A, Tejedor L 2013: Outcome of surgical resection in Klatskin tumors. World J Gastrointest Oncol 5: $147-158$

Srimathveeravalli G, Wimmer T, Monette S, Gutta NB, Ezell PC, Maybody M, Weiser MR, Solomon SB 2013: Evaluation of an endorectal electrode for performing focused irreversible electroporation ablations in the swine rectum. J Vasc Interv Radiol JVIR 24: 1249-1256

Srimathveeravalli G, Silk M, Wimmer T, Monette S, Kimm S, Maybody M, Solomon SB, Coleman J, Durack JC 2015: Feasibility of catheter-directed intraluminal irreversible electroporation of porcine ureter and acute outcomes in response to increasing energy delivery. J Vasc Interv Radiol 26: 1059-1066

Topazian M, Levy MJ, Patel S, Charlton MR, Baron TH 2013: Hepatic artery pseudoaneurysm formation following intraductal biliary radiofrequency ablation. Endoscopy 45 Suppl 2 UCTN: E161-162

Ueshima E, Schattner M, Mendelsohn R, Gerdes H, Monette S, Takaki H, Durack JC, Solomon SB, Srimathveeravalli G 2018: Transmural ablation of the normal porcine common bile duct with catheter-directed irreversible electroporation is feasible and does not affect duct patency. Gastrointest Endosc 87: 300.e1-300.e6

Zhang Y, Guo Y, Ragin AB, Lewandowski RJ, Yang G-Y, Nijm GM, Sahakian AV, Omary RA, Larson AC 2010: MR imaging to assess immediate response to irreversible electroporation for targeted ablation of liver tissues: preclinical feasibility studies in a rodent model. Radiology 256: 424-432 


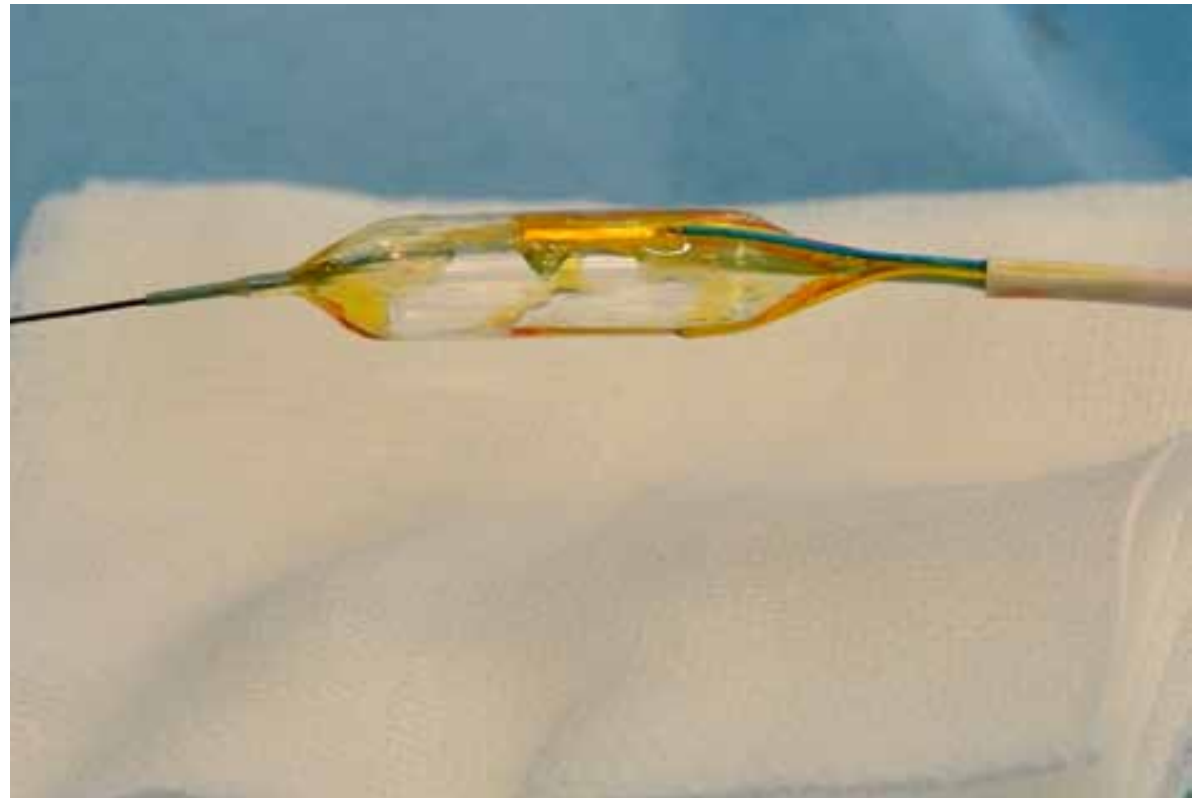

Fig. 1. Prototype of endoluminal catheter for irreversible electroporation

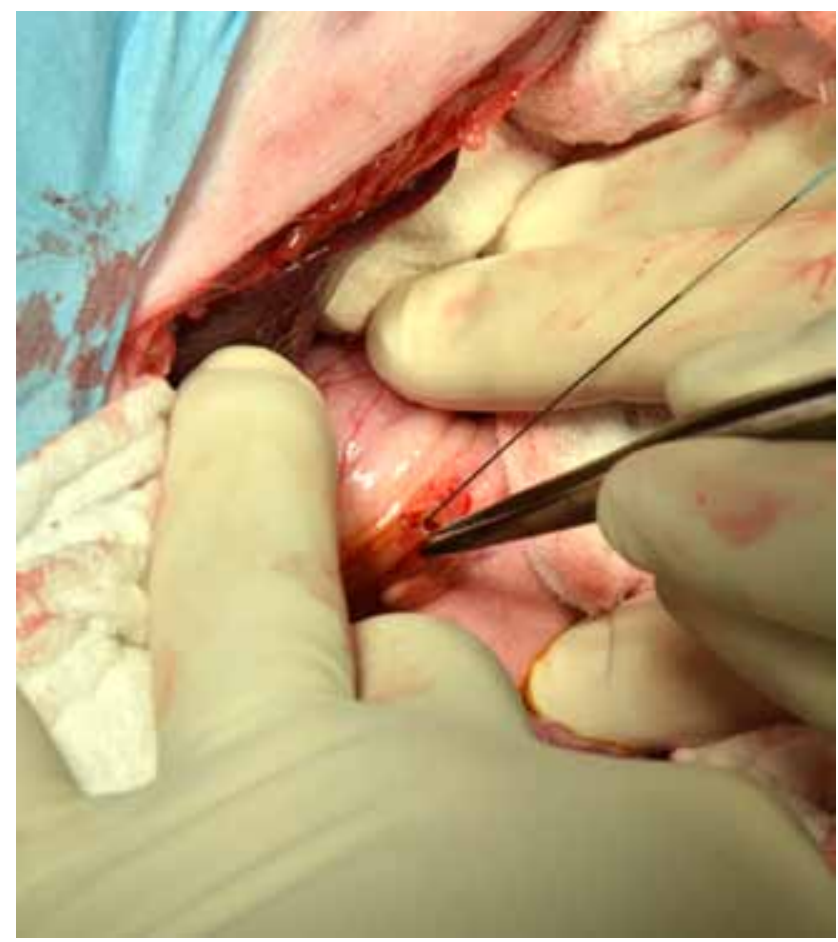

Fig. 2. Insertion of guidewire to the distal part of the common bile duct 


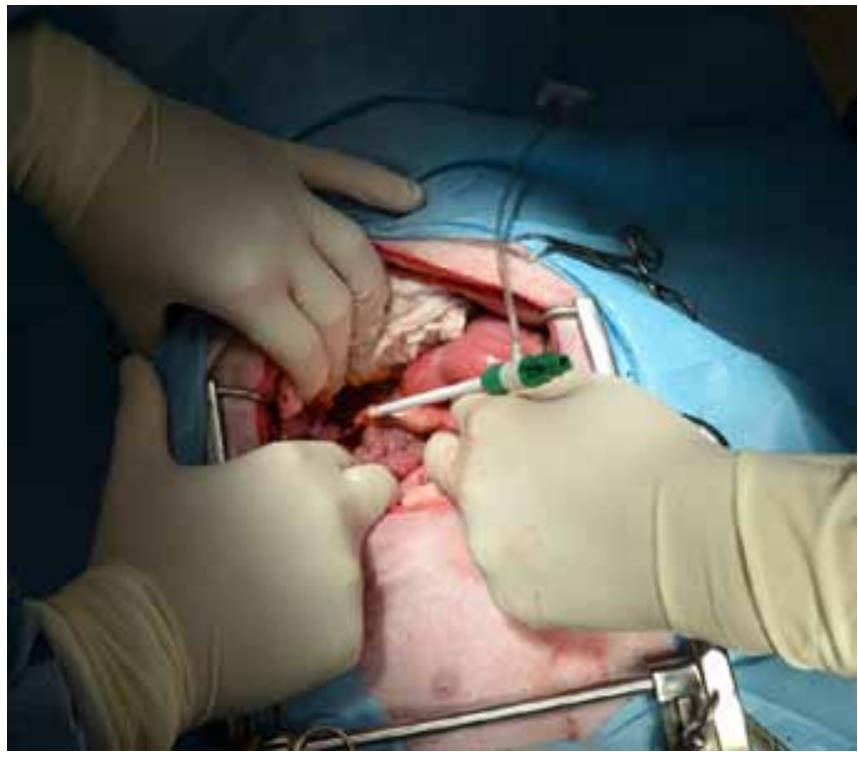

Fig. 3. Insertion of $10 \mathrm{~F}$ sheath to the common bile duct.

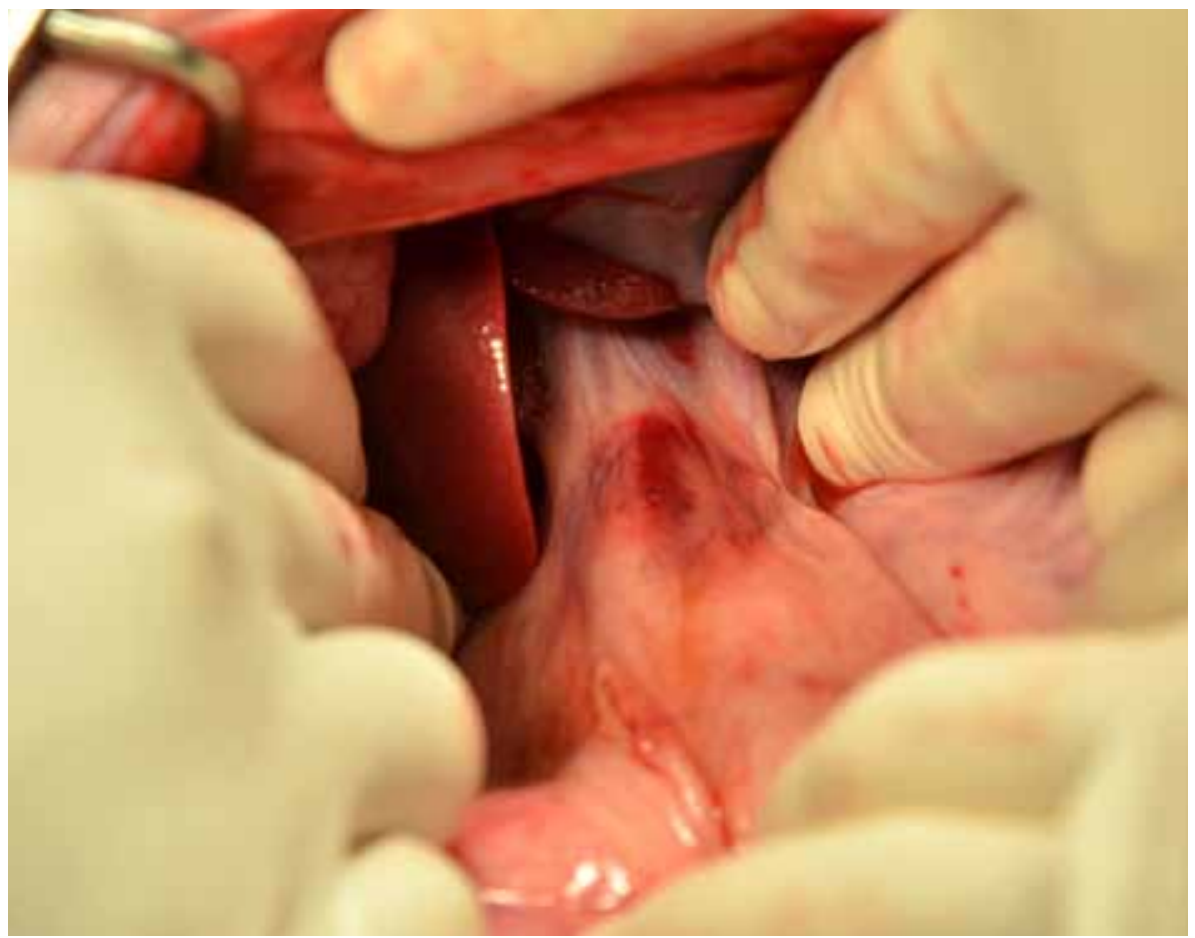

Fig. 4. Macroscopic changes immediately after irreversible electroporation in the common bile duct (red area of focal haemorrhage) 


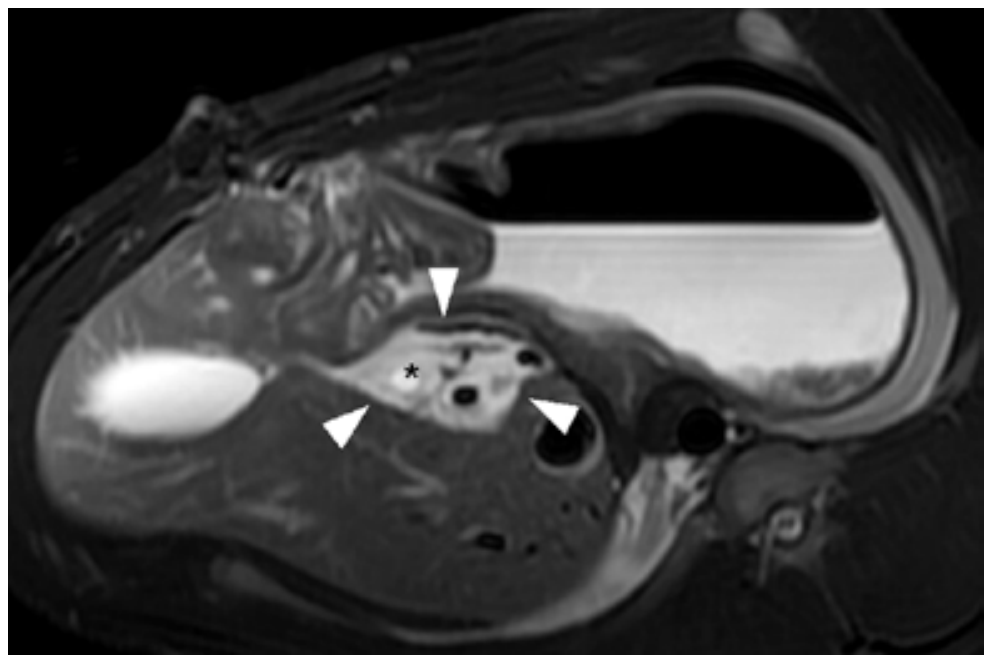

Fig. 5. Postprocedural T2 weighted image (T2 FR FSE) shows oedema in the hepatoduodenal ligament (hyperintensity, arrowheads) surrounding the common bile duct (asterisk)

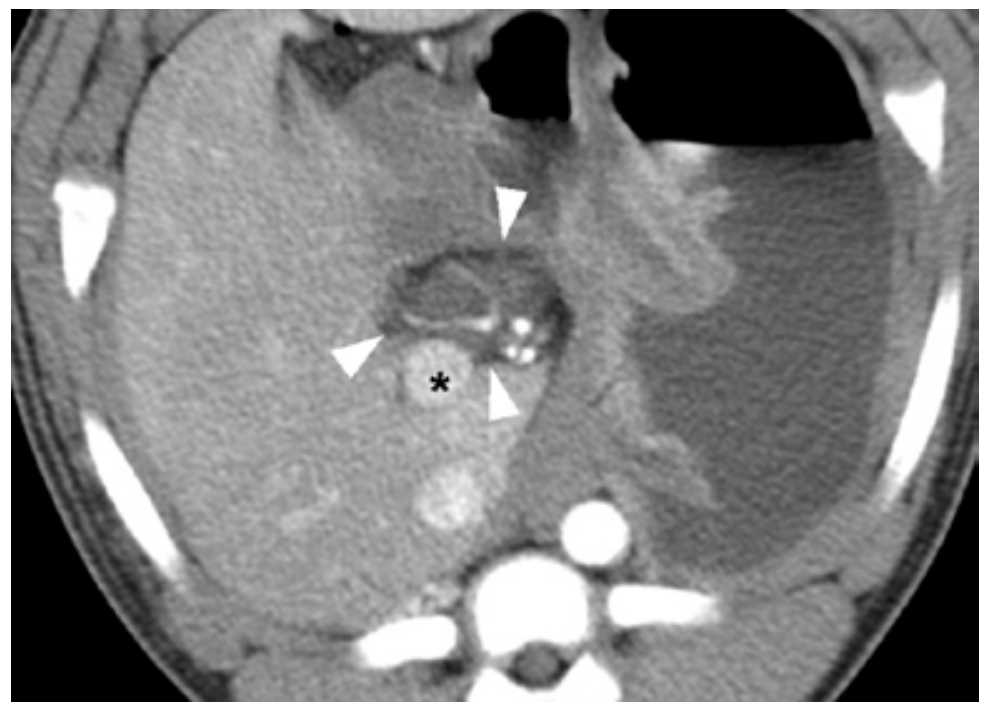

Fig. 6. Multidetector computed tomography image with contrast agent $24 \mathrm{~h}$ after surgery demonstrates oedema in the hepatoduodenal ligament (marked hypodensity, arrowheads) and patent vascular structures (hepatic artery, portal vein - asterisk) 


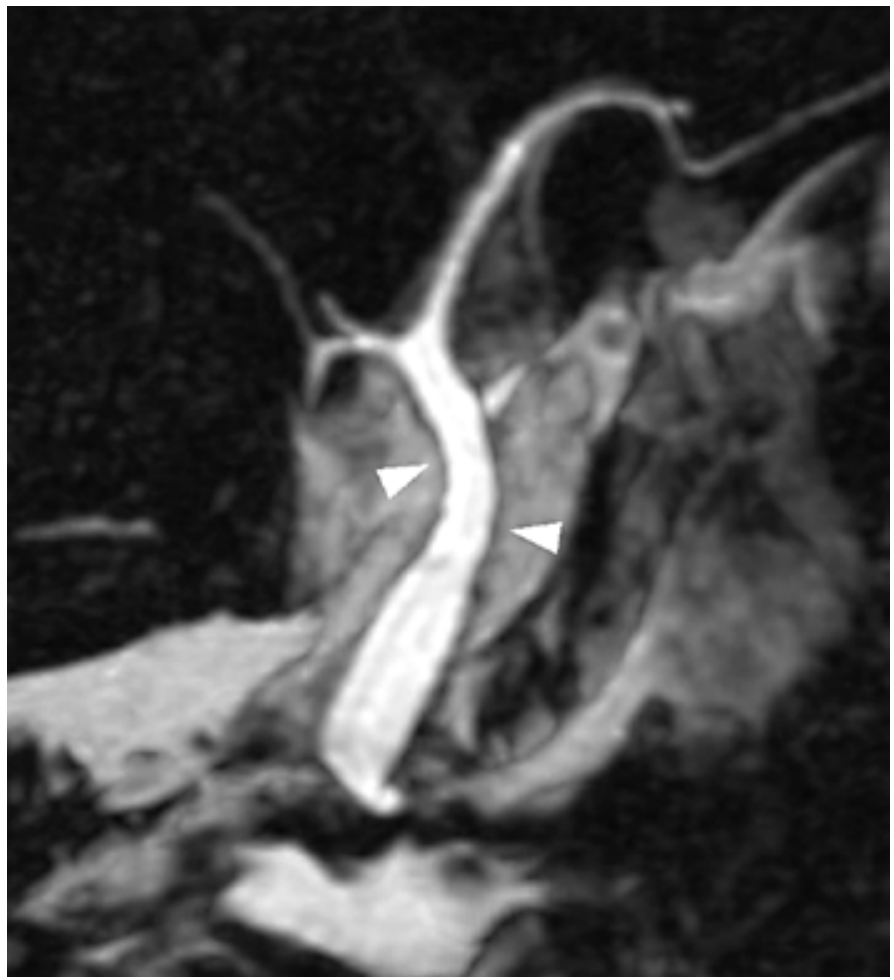

Fig. 7. Magnetic resonance cholangiopancreatography revealed the site of irreversible electroporation as a minor tapering of the common bile duct (arrowheads) and surrounding hyperintensities due to oedema of tissue

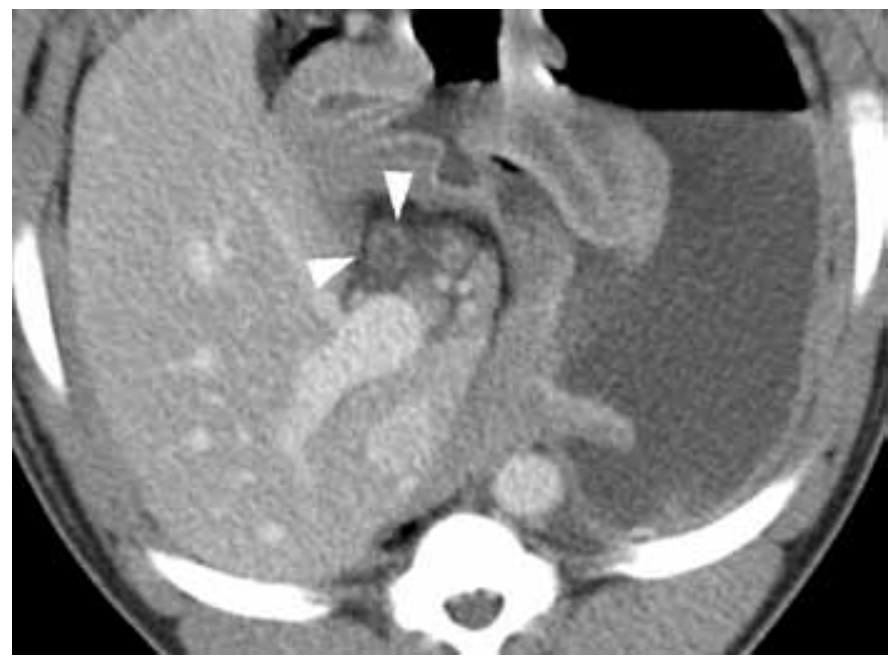

Fig. 8. Multidetector computed tomography image with contrast agent $96 \mathrm{~h}$ after procedure shows faint contrast enhancement of the common bile duct wall (arrowheads) at the site of ablation and regular enhancement of adjacent vessels 


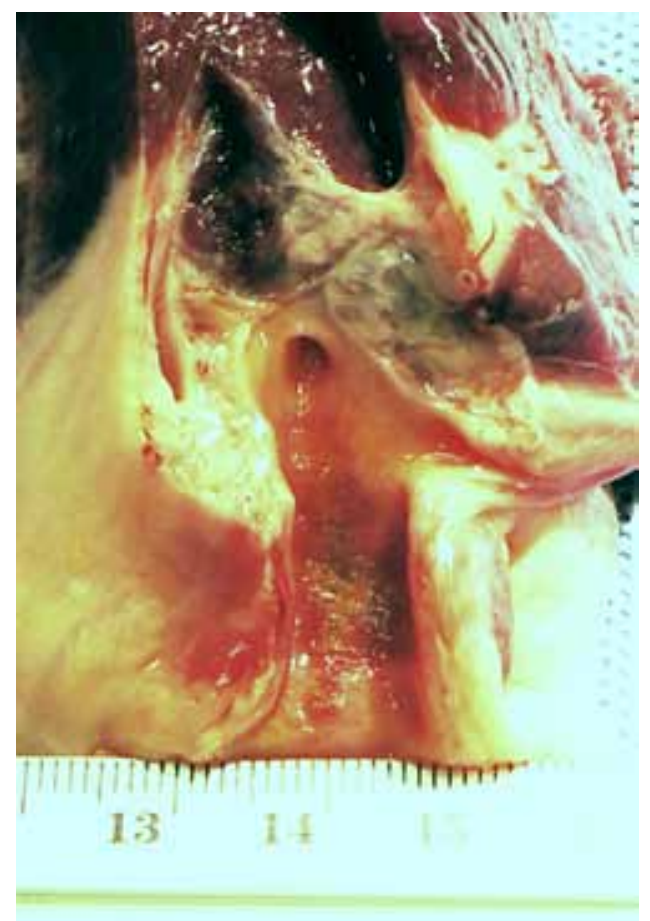

Fig. 9. Gross changes $24 \mathrm{~h}$ after procedure, well demarcated zone of irreversible electroporation is visible

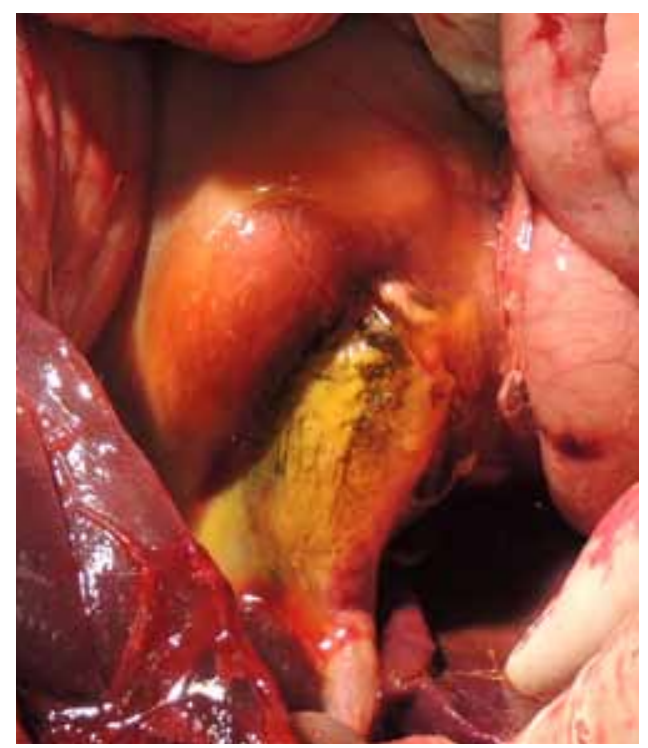

Fig. 10. Covered perforation of the common bile duct with bilioma formation in the hepatoduodenal ligament and emphysema 


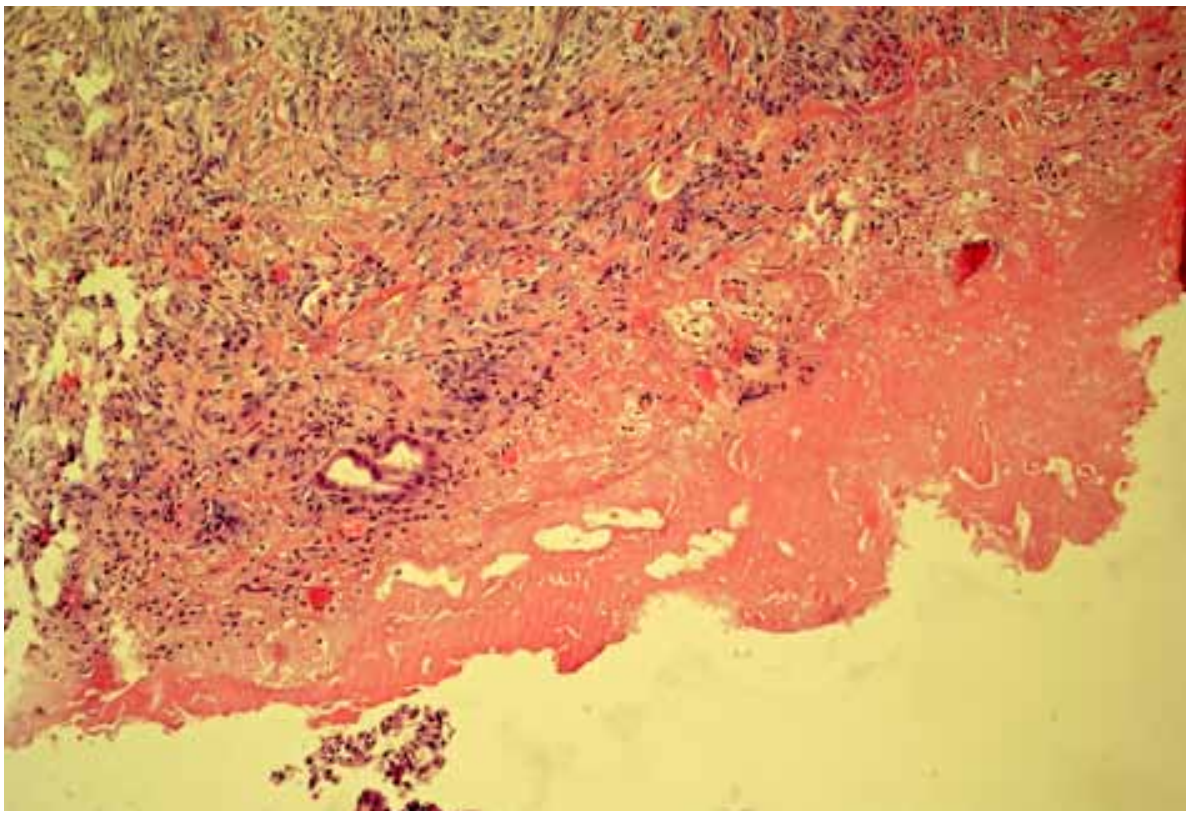

Fig. 11. Haematoxylin and eosin staining, $\times 100$. Necrosis of the surface epithelium and lamina propria of the proximal common bile duct wall with proliferation of granulation tissue below the ulceration.

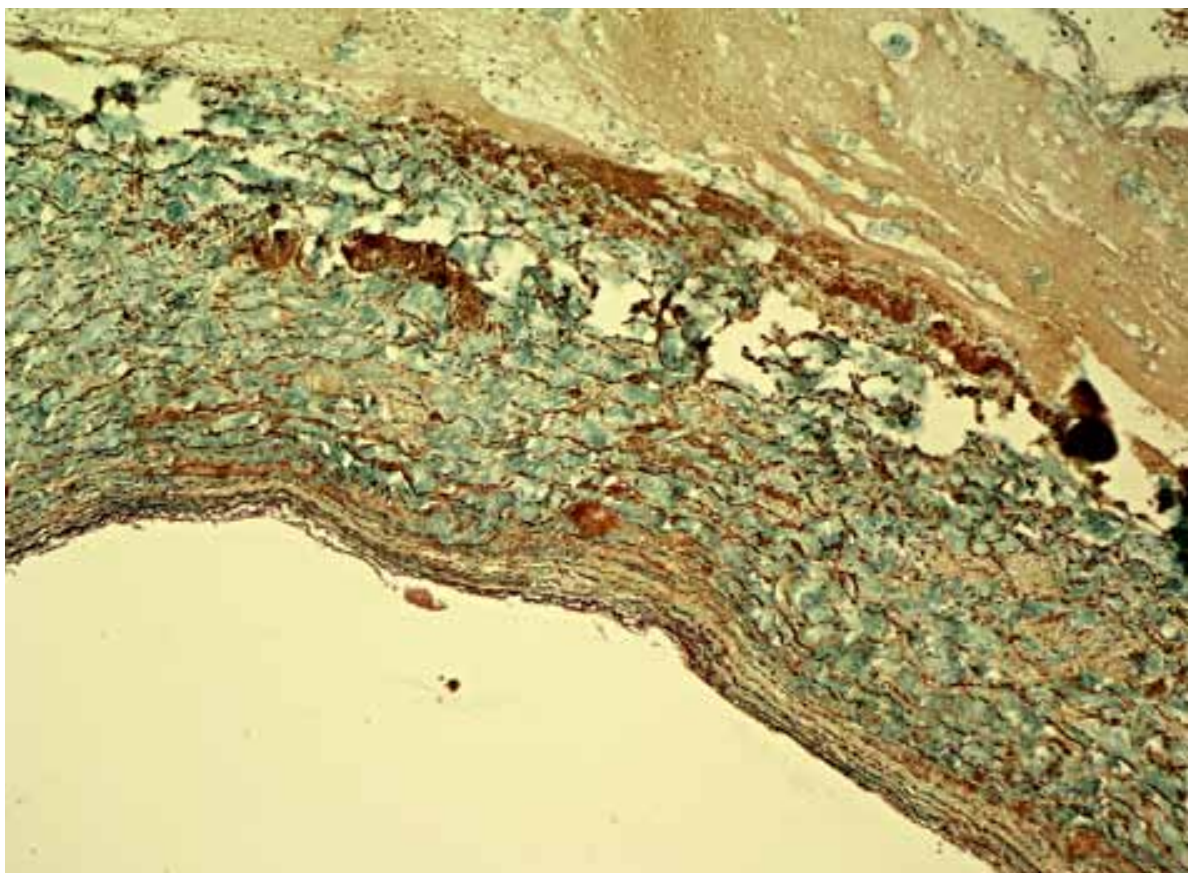

Fig. 12. Orcein staining, $\times 100$. The elastic membranes of portal vein are preserved while endothelial cells are necrotic. 